\title{
PASOAC-Net: A Petri-Net Model to Manage Authorization in Service-Based Business Process
}

\author{
Haiyang Sun ${ }^{1}$, Weiliang Zhao ${ }^{2}$, and Surya Nepal ${ }^{3}$ \\ 1 Department of Computing, Macquarie University, Sydney, Australia \\ 2 Faculty of Engineering, University of Wollongong, Wollongong, Australia \\ 3 CSIRO ICT Centre, Sydney, Australia \\ haiyang.sun@mq.edu.au, wzhao@uow.edu.au, Surya.Nepal@csiro.au
}

\begin{abstract}
A successful execution of a Business Process (BP) is possible only if the proper coordination exists between (1) BP's execution policy, (2) BP's authorization policy, and (3) the authorization policies of BP's resources. Hence, there is a need of an effective authorization model that brings all types of policies together for a BP executing successfully without breaking any authorization and business rules. This paper proposes a Petri-Net process model, Process-Aware Service-Oriented Authorization Control Net (PASOAC-Net). PASOAC-Net is developed based on the conceptual model PASOAC, an extension of Role Based Access Control (RBAC), which takes both resources and users into account. A set of authorization constraints is designed in PASOAC to coordinate the user access and the resource support in a process environment.
\end{abstract}

\section{Introduction}

For a successful execution, a Business Process (BP) must be able to satisfy authorization policies of resources to receive their supports. The interactions between the BP and its users are also imperative. Users need to satisfy the process authorization policies before accessing to the specific process functions. Execution policies are used to manage the sequence of task invocations within a BP, i.e., business logic. Without an appropriate coordination on these policies, a BP may not be able to perform properly. Therefore, how to manage the user accesses and the resource supports in a BP in a distributed environment, e.g., web service domain, becomes a challenging task. We will discuss this point further through a motivating example.

\subsection{Motivating Example}

In Fig. 1, we illustrate an execution sequence of Financial Lease BP. Each operation (also known as task) of BP is depicted as a rectangle in the process. A User table is used to illustrate three types of users, and their associated access policies on operations. Four types of resources are illustrated in Resource table in Fig. 1, The sequence of interactions among users, resources, and operations of $\mathrm{BP}$ is numbered in Fig. 1, The requirements for accessing and supporting Financial Lease are stated below, 


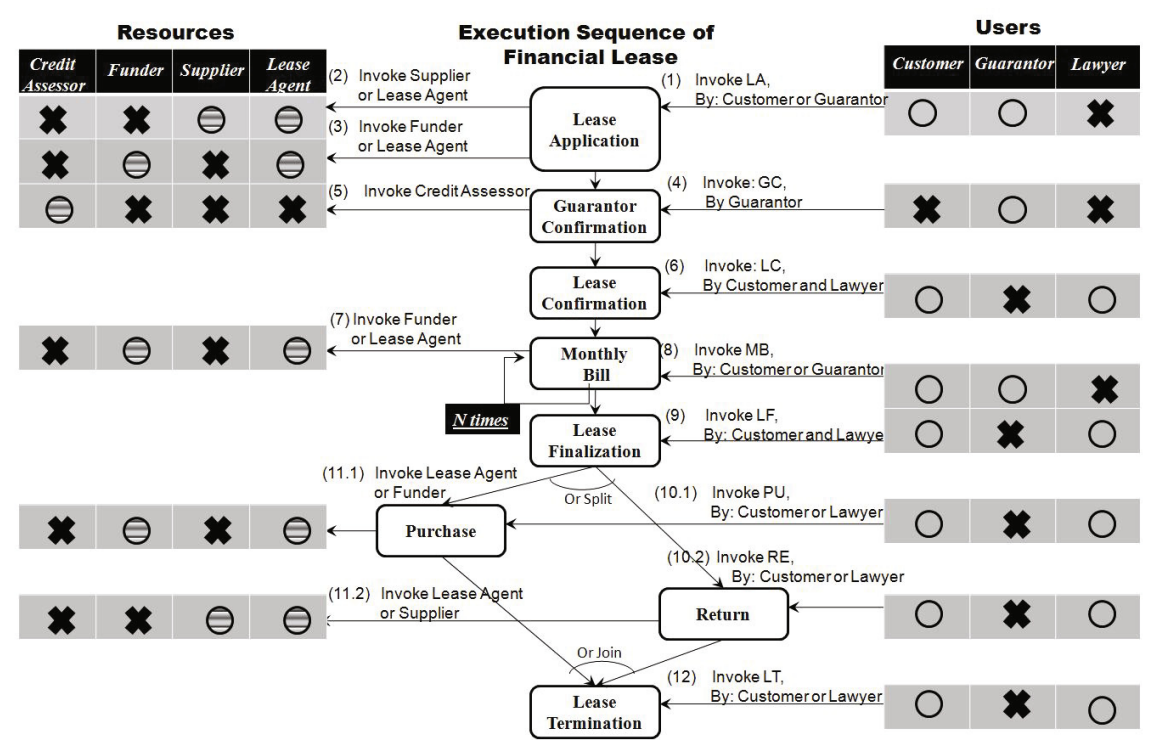

Fig. 1. Execution Sequence of Financial Lease

- User: (1) Customer should be able to access all operations. (2) The operation Guarantor Confirmation can only be made by Guarantor. Guarantor can start the lease on behalf of the Customer and help to repay the rental. (3) Lawyer after lease finalization stage can deal with any rest activities on behalf of its client. Furthermore, Lawyer is necessary to involve in operations of Lease Confirmation and Lease Finalization with Customer.

- Resource: (1) Funder is used to provide financial support. (2) Supplier is the product provider. (3) Lease Agent can provide both product and fund to Financial Lease, since the Lease Agent will seek its own Funder and Supplier. (4) Credit Assessor can evaluate the credit histories of Guarantor and Customer.

We can observe from the above example that the user accesses and the resource supports are not only regulated by their specific authorization policies, but also need to be restricted by the business constraints enacted during the execution of BP. Otherwise, authorization issues can be raised to cease process execution. These business constraints can be categorized as follows,

- Synchronization: The sequence of the user accesses and resource supports should be synchronized with the execution sequence of the operations in BP. When an operation is ready to execute in a process instance, the relevant users and resources that can access and support the operation should be invoked. Once the operation finishes, the permissions assigned to the user and resource to access and support the operation should be revoked immediately. For example, when the operation Guarantor Confirmation starts, 
the authorization to access the operation should be granted to Guarantor. When the operation finishes, the authorization of Guarantor access to the operation should be revoked immediately to avoid repeated submissions of guarantor information.

- Dependency: A user access of (or a resource support on) a specific operation in a BP may depend on another user access (or resource support). In the above example, operation Monthly Bill can be supported by Funder or Lease Agent and accessed by Customer or Guarantor. To avoid fraudulent activity, a Guarantor can pay the rental on behalf of the Customer to access the operation Monthly Bill only if a Funder issues the bill. This constraint is used when an entity can play as both Lease Agent and Guarantor. Obviously, if the entity pays the bill issued by itself, it may eventually do harm to Customer's interest.

Therefore, an effective authorization model is highly desirable to support the coordination of the user accesses and resource supports in a BP. In this work, a conceptual model named Process-Aware Service-Oriented Authorization Control (PASOAC) is proposed to manage authorization of business process by considering both user access and resource support. Two types of authorization policies, (1) Authorization Synchronization Policies and (2) Authorization Dependence Policies, are included in the conceptual model to deal with the above business security requirements. Authorization flow (i.e, sequences of user accesses and resources supports with associated authorization policies) is modeled by a PetriNet based process model PASOAC-Net.

The rest of paper is organized as follows. Section 2 describes the conceptual model PASOAC. Authorization policies are also defined in this section. The specification of PASOAC-Net is described in Section 3. Section 4 overviews some related work. Concluding remarks are provided in Section 5.

\section{Conceptual Model-PASOAC}

In this section, we specify the PASOAC conceptual model by using the notation of Entity-Relationship (E-R) Diagram. In Fig. 2, rectangles represent elements and diamonds represent relationships. We define Role $(\mathbf{R})$ as a type of user that requires to access the operations $(\mathbf{O p})$ of BP. Resource type $(\mathbf{R e T})$ is defined as a type of resource that can provide support on the operation $(\mathbf{O p})$. Their associated relationships, access and support, are all many-to-many.

In Fig. 2 there are two types of authorization policies, Synchronization and Dependency. A sequence of role accesses on operations of BP can be called as role-flow; while a sequence of resource type support on operations of BP can be called as resource type-flow. An authorization flow consists of role-flow and resource type-flow, and must be executed consistently with the control flow of BP. In Fig. 2, three red arrows are used to represent the sequence of role access (role-flow), business logic of BP (control-flow), and the sequence of resource type support (resource type-flow) respectively. Authorization Synchronization Policy 


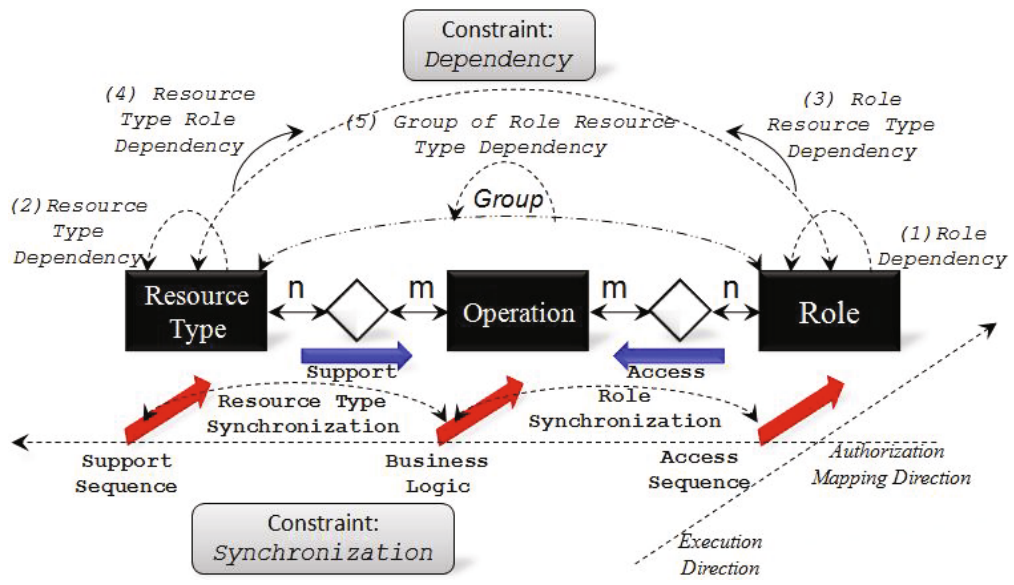

Fig. 2. Process-Aware Service-Oriented Authorization Control

is therefore divided into two types, (1) Role Synchronization Policy and (2) Resource Type Synchronization Policy. Authorization Dependency Policies restrict that a role or a resource type is not able to access or support the operations, until the other role or the other resource type has already accessed or supported specific operations. Authorization Dependency Policies are separated into 5 categories, (1) between roles, (2) between resource types, (3) between roles and resource types, (4) between resource types and roles, and (5) between groups of role and resource type (See Fig. 2).

\section{Specification of PASOAC-Net}

PASOAC-Net is a Petri-Net process model divided into three parts, role-net, resource type-net, and constraint-net, respectively.

\subsection{Structure of PASOAC-Net}

Role-Net is used to model role-flow and enforce the Role Synchronization Policy. In role-net, we use each transition (the black rectangle) to represent a role access on specific operation. We use token that flows between the transitions in role-net to represent the operations. When the transition consumes one token, it means that the operation (token) has been accessed by the role (the transition). The new generated tokens by this transition will represent the next operation that need to be accessed according to the business logic of BP. Role-Net is formally defined as follows,

Definition 1 Role-Net is a tuple $\mathcal{H}=\left(P^{r}, T^{r}, F^{r}, i^{r}, o^{r}\right)$ 
- $P^{r}$ is a set of places, graphically represented as circles,

- $T^{r}$ is a set of transitions, graphically represented as black bars to represent role accesses on specific operations in role-net. $T^{e} \subset T^{r}$ is a set of empty transitions as or-split, or-join, and-split, or and-join,

- $F^{r}=\left(P^{r} \times T^{r}\right) \cup\left(T^{r} \times P^{r}\right)$,

- $i^{r}$ and $o^{r}$ are input place and output place respectively, to initially deposit token and finally collect token in role-net.

Resource Type-Net is used to model resource type-flow and enforce the Resource Type Synchronization Policy. It bears the similar semantics as RoleNet. The formal definition of resource type-net is defined as follows,

Definition 2 Resource Type-Net is a tuple $\mathcal{G}=\left(P^{\text {ret }}, T^{\text {ret }}, F^{r e t}\right.$, $i^{\text {ret }}$, oret $)$,

- $P^{r e t}$ is a set of places graphically represented as circles,

- $T^{\text {ret }}$ is a set of transitions, graphically represented as black bars to represent resource type supports in resource type-net. $T^{e} \subset T^{r e t}$ is a set of empty transitions as or-split, or-join, and-split, or and-join,

- $F^{r e t}=\left(P^{r e t} \times T^{r e t}\right) \cup\left(T^{r e t} \times P^{r e t}\right)$,

- $i^{\text {ret }}$ and $o^{\text {ret }}$ are input place and output place respectively, to initially deposit token and finally collect token in resource type-net.

Constraints-Net is used to enforce the five Authorization Dependency Policies. Each transition in constraint-net is linked from one transition in role-net or resource type-net and points to another transition in role-net or resource typenet. Hence, without token movement through the transition in constraints-net, the relevant role-net or resource type-net which is pointed by the transition in constraints-net can not accumulate enough tokens to fire, according to the basic execution policy of Petri-Net. We can use this method to realize the dependency between roles, between resource types, even between role and resource type. The group dependency policy is different from the other dependency polices, in that the depending role and resource types can be executed individually, but can not be both executed if the depended role and resource type are not both executed. The formal definition of constraint-net is defined as follows,

Definition 3 Constraints-Net is a tuple $\mathcal{C}=\left(P^{c}, T^{c}, F^{c}, W\right.$, Count $)$, Where:

- $P^{c}$ is a set of places graphically represented as circles,

- $T^{c}$ is a set of transitions, graphically represented as black bars to represent constraints in between roles, between resource types, and between role and resource type. For group dependency policy, transitions are separated as follows,

$-T^{e} \subset T^{c}$ is a set of empty transitions as and-split to simultaneously split the token movement pathes to $T_{x} \subset T^{c}$ and $T_{y} \subset T^{c}$.

- $T_{x} \subset T^{c}$ represents an indictor to show, when Group $B$ depends Group A, if a role or a resource type in group $B$ has already been used to deal with specific operation.

- $T_{y} \subset T^{c}$ represents an indictor to show if both role and resource type in group A have been executed. 
- $F^{c}=\left(P^{c} \times T^{c}\right) \cup\left(T^{c} \times P^{c}\right) \cup\left(T^{r e t} \times P^{c}\right) \cup\left(P^{c} \times T^{r e t}\right) \cup\left(T^{r} \times P^{c}\right) \cup\left(P^{c} \times T^{r}\right)$, where, $T_{x} \bullet \times T^{r}$ and $T_{x} \bullet \times T^{r e t}\left(T_{x} \bullet=\left\{p \in P^{c} \mid\left(T_{x} \times p\right) \in F^{c}\right\}\right)$ can be weak relation or normal relation depending on the amount of tokens in $T_{x} \bullet$. (1) If no token is deposited in $T_{x} \bullet$, then the relation between $T_{x} \bullet$ and the transition in role-net or resource type-net becomes a weak relation that will not affect the execution of the linked transition. (2) When at least one token is deposited in $T_{x} \bullet$, the relation between $T_{x} \bullet$ and the transition in role-net or resource type-net becomes normal relation that affects the execution of the linked transition.

- $W: F^{c} \rightarrow I N$ is a weight function on the relation between place and transition. It reflects how many tokens are needed to pass the relation (IN represents Integer $)$. For example, $W:\left(\bullet T_{y}, T_{y}\right) \rightarrow 2\left(\bullet T_{y}=\left\{p \in P^{c} \mid\left(p, T_{y}\right) \in F^{c}\right\}\right), W:\left(T_{x} \bullet\right.$, $\left.T_{r}\right) \rightarrow 2$ and $W:\left(T_{x} \bullet, T_{r e t}\right) \rightarrow 2$. The weights of all other relations are 1.

- Count: $P^{c} \rightarrow I N$ is a function to calculate the amount of tokens at each place. The result of this function on specific place can be used to decide if the relation between transition and this place is weak relation or normal relation.

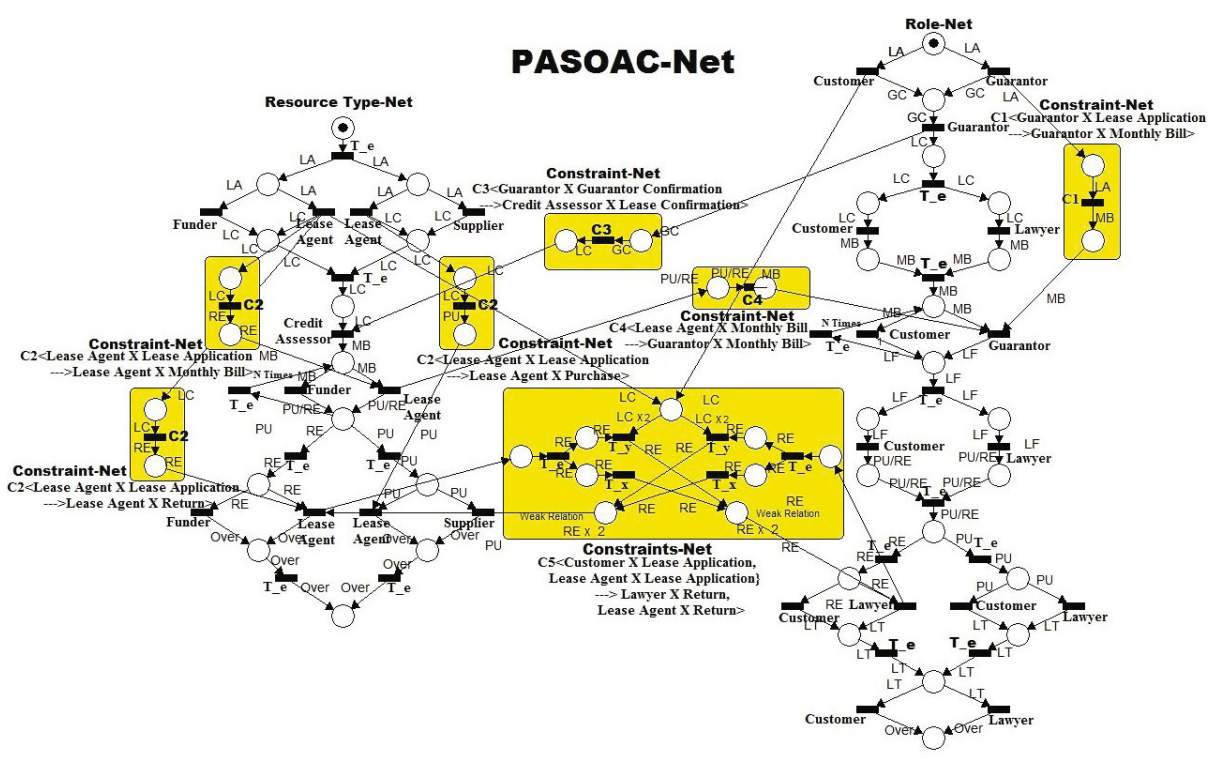

Fig. 3. PASOAC-Net

In Fig. 3. a comprehensive view of PASOAC-Net based on the motivating example is presented, where both role-net and resource type-net are illustrated as well as each category of constraint-net. 


\subsection{Execution of PASOAC-Net}

The token movement in PASOAC-Net is regulated by its execution mechanism. The PASOAC-Net execution complies with the general execution policy of PetriNet. At beginning, two tokens are deposited at each initial place of role-net and resource type-net. Once the tokens reach the final place of each net, we believe that the BP is successfully executed by complying with all authorization policies.

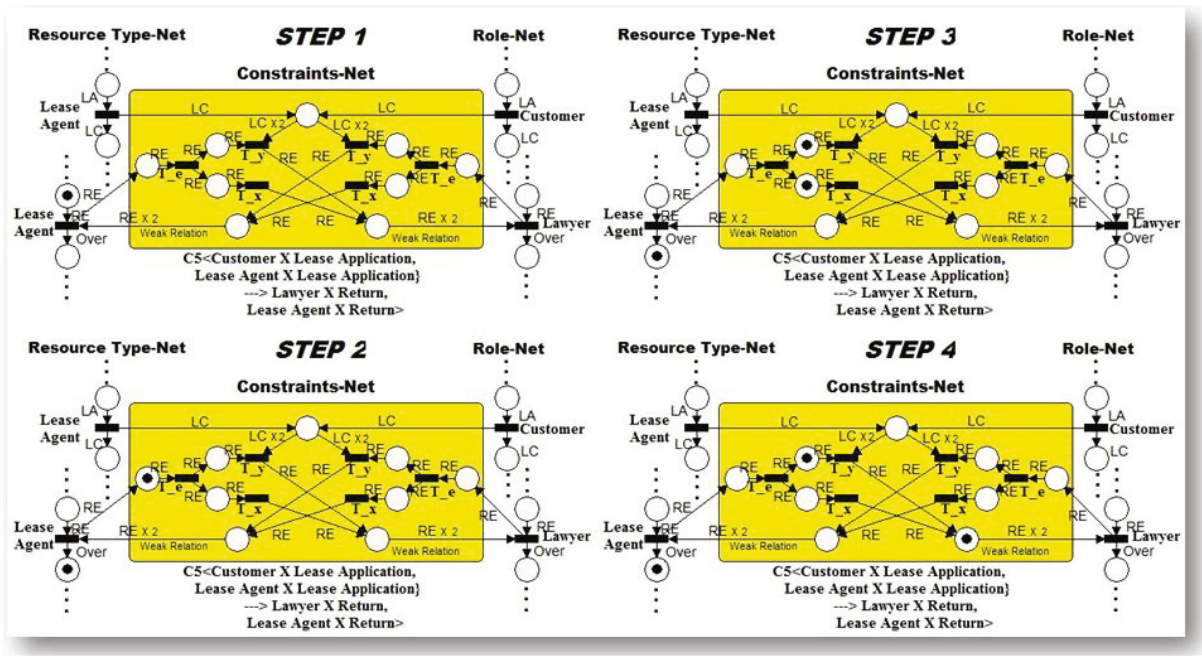

Fig. 4. Execution Mechanism of Constraint-Net for Group Dependency Policy

However, the execution mechanism designed for group dependency policy is complicated that introduces in details here. For example, in Fig. 4, the group of Lease Agent support and Lawyer access on the operation Return depends on the group of Lease Agent support and Customer access on the operation Lease Application. Initially, only the transition (Lease Agent support on Return) in resource type-net is enabled by a token arrival in its pre-place. There is no token deposited in $T_{x} \bullet$ in constraint-net. The relation between $T_{x} \bullet$ and the transition (Lease Agent support on Return) is weak relation, that will not affect the fire of this transition (See Step 1 in Fig. 4). After firing, one token is passed to $T_{e}$ in constraint-net and the other one is moved into the post-place of this transition in resource type-net (See Step 2 in Fig. 4). The token in pre-place of $T_{e}$ in constraint-net is split into $\bullet T_{y}$ and $\bullet T_{x}$ after $T_{e}$ firing (See Step $\mathbf{3}$ in Fig. 4). At this time, $T_{x}$ can be enabled and fired to deposit one token from $\bullet T_{x}$ to $T_{x} \bullet$. When Count $\left(T_{x} \bullet\right)=1$, the relation between $T_{x} \bullet$ and the transition (Lawyer access on Return) in role-net becomes normal relation that affects the fire of the transition. Since $\mathrm{W}: T_{x} \bullet \times T_{r}=2$ and $\mathrm{W}: T_{x} \bullet \times T_{\text {ret }}=2$, one token in $T_{x} \bullet$ now is not enough to enable the normal relation with weight 2 . Therefore, even if the preplace of the transition (Lawyer access on Return) in role-net has accumulated 
enough token, the transition still can not be fired, since its pre-place $\left(T_{x} \bullet\right)$ in constraints-net has not accumulated enough tokens to pass the relation with weight 2 . The group dependency policy is enforced to restrict the Lawyer access on Return before the group of Lease Agent support and Customer access on the operation Lease Application have both been executed (See Step 4 in Fig. 4).

\section{Related Work}

Role based access control (RBAC) [1, 2, 3, is a widely accepted approach on $\mathrm{BP}$ authorization. In 4, the authors proposed a workflow authorization model (WAM) and an authorization template (AT) to realize the synchronization of role-flow with workflow. In [5], the authors propose a constrained workflow system where local and global cardinality constraints as well as SoD and BoD are enforced. However, all above authorization models in workflow environment, that deal with resources within the same security domain as workflow, can not be used directly as ready solutions for authorization of service based BP, since resources in web service domain spread across organizational boundary. The authorization dependency policies are also missing in the existing models.

\section{Conclusion and Future Work}

In this paper, we propose an authorization conceptual model PASOAC for managing user access and resource support in business processes. Two types of authorization constraints, Synchronization and Dependency, are considered in PASOAC. A Petri-Net based process model PASOAC-Net is developed based on PASOAC as a formal infrastructure to ensure the successful execution of BP by enforcing various types of synchronization and dependency policies.

\section{References}

[1] Sandhu, R.S., Coyne, E., Feinstein, H., Youman, C.: Role-based Access Control Models. IEEE Computer 29(2), 38-47 (1996)

[2] Ahn, G., Sandhu, R.: Role-Based Authorization Constraints Specification. ACM Transactions on Information and System Security (TISSEC) 3(4), 207-226 (2000)

[3] Ferraiolo, D., Sandhu, R., et al.: Proposed NIST Standard for Role-Based Access Control. TISSEC 4(3), 224-274 (2001)

[4] Atluri, V., Huang, W.-K.: An Authorization Model for Workflows. In: Martella, G., Kurth, H., Montolivo, E., Bertino, E. (eds.) ESORICS 1996. LNCS, vol. 1146, pp. 44-64. Springer, Heidelberg (1996)

[5] Tan, K., Crampton, J., Gunter, C.A.: The consistency of task-based authorization constraints in workflow. In: IEEE Workshop of Comp. Security Foundations (2004)

[6] Wonohoesodo, R., Tari, Z.: A Role Based Access Control for Web Services. In: Proceedings of SCC, pp. 49-56 (2004)

[7] Fischer, J., Majumdar, R.: A Theorey of Role Composition. In: Proceedings of ICWS, pp. 49-56 (2008) 